\title{
INFLUENCE OF SURFACE IRREGULARITIES ON HYDRODYNAMIC INSTABILITIES IN COUETTE-TAYLOR FLOW
}

\author{
LAMIA GAIED, MARC LIPPERT, LAURENT KEIRSBULCK, MAXENCE BIGERELLE, FETHI \\ ALOUI
}

\author{
UNIV. VALENCIENNES, UMR 8201 - LAMIH - LABORATOIRE D'AUTOMATIQUE DE \\ MÉCANIQUE ET D'INFORMATIQUE INDUSTRIELLES ET HUMAINES, F-59313 \\ VALENCIENNES, FRANCE B CNRS, UMR 8201, F-59313 VALENCIENNES, FRANCE
}

\section{EMNA BERRICH BETOUCHE}

\section{LUNAM UNIVERSITY, NANTES UNIVERSITY, CNRS, GEPEA, UMR 6144, IMT ATLANTIQUE, DSEE, 4 RUE ALFRED KASTLER - BP20722 44307 NANTES CEDEX 03 - FRANCE}

\section{ABSTRACT}

In this work, we propose an experimental study on the hydrodynamic instabilities due to the roughness of the wall in a Couette-Taylor flow. The study was carried out without an axial flow. In our experiments, the inner cylinder rotates at a certain angular velocity and the outer cylinder is maintained fixed.

The main objective of the study is to highlight the geometric parameters effect on the flow of the flow. We mainly focused on the effect of roughness of the wall on the appearance of instabilities in the studied configuration (delay or advance in its appearance). For this purpose, a qualitative study was performed using Kalliroscope particles to visualize the instabilities. Then, we applied polarography to different types of roughness as a quantitative study. Hence the possibility to characterize the flow regimes of the first instabilities (TVF, $W V F, M W V F$, and TN).

Our experience has shown that surface irregularities, actually, have an effect on the appearance of the first instabilities depending on the size and nature of the roughness. In fact, the surface roughness not only increases the friction on the wall but it also greatly influences the transport of the momentum and mass in a given flow. The flow, therefore, undergoes more friction when the inner cylinder (in rotation) has a rough surface. This friction slows the velocity of the fluid particles; which delays the onset of instability. The movement of the particles will therefore, be dampened by the irregularities at the level of the rough surface.

Moreover, the results also proved that as soon as the Taylor cells are formed, the roughness is no longer a particle motion damper, but on the contrary, it promotes the continuation of the disturbance of the flow. The flow then becomes less slow down in the hollow of the surface irregularities; which leads to less friction. For higher Taylor numbers, the study showed a faster transition from one regime to another.

Keywords: Taylor-Couette Flow, Surface Roughness, Rectangular groove, Hydrodynamic instabilities.

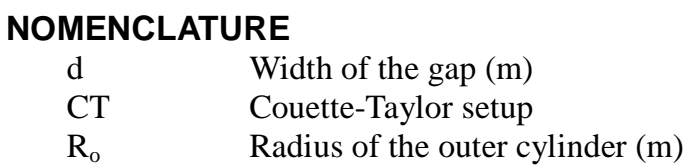




\section{$\mathrm{R}_{\mathrm{i}} \quad$ Radius of the inner (grooved) cylinder (m) \\ $\mathrm{H} \quad$ Height of the two cylinders (m) \\ Sh Sherwood number \\ Ta Taylor number \\ $\eta \quad$ Radial ratio \\ $v \quad$ Kinematic viscosity $\left(\mathrm{m}^{2} / \mathrm{s}\right)$ \\ $\Gamma \quad$ Axial aspect ratio \\ $\Omega \quad$ Angular velocity $(\mathrm{rd} / \mathrm{s})$ \\ I. INTRODUCTION}

The study of the flow of a fluid around a solid body with a rough or structured surface occupies a considerable interest as it is encountered in the verification of reliability and the performance of rotating machines (turbines or hydrodynamic bearings). The design and modeling of these machines with a high degree of performance is one of the associated applications.

This configuration is analogous to the configuration known as Couette Taylor flow, a flow between two concentric cylinders: the outer cylinder is fixed and the inner cylinder is rotatable. An axial flow is also taken into account so the flow is called Couette Taylor-Poiseuille flow.

Many studies have been done on the thermal part (heat transfer) for configurations where the inner cylinder is smooth. In the case where the cylinders of the system have roughness, some scientists are focused their studies mainly on the heat transfer.

For the specific problem of rotating machines (rotors) with non-smooth surfaces, the study of phenomena in the gap between the two cylinders becomes a major problem to optimize their performance.

A better understanding of the characteristics of the flow field in this confined gap still requires specific deep studies. This is necessary in order to improve their design and develop more efficient models.

Since Couette's studies [1], Couette-Taylor flow has influenced many scientists, experimentally, numerically and theoretically. Up to now, several experimental, theoretical and recent numerical studies have been dedicated to this problem, and particularly to the Taylor vortices [2-6].

Regarding the thermal part, most studies reported the case of smooth cylinders. Only a few studies include references to an axially grooved cylinder.

Among the experimental studies, mention is made of Cadot et al. [7] and Van den Berg et al. [8] who are interested in the study of Taylor quilt system having ribs in the form of square or triangular regularly distributed on the surface of cylinders, at large Reynolds numbers $\left(\operatorname{Re}_{\mathrm{i}}=3 \times 10^{5}\right)$.

In these references $[7,8]$, the most interesting conclusion is that in the case where the system consists of two rough cylinders, the drag coefficient is independent of the Reynolds number.

The authors noted that in the case of smooth cylinders, the total dissipation is thus dominated by the contribution of the boundary layers, whereas it is dominated by the contribution of mass transfer for the case of the striated cylinders.

Numerically, Tsukahara et al. [9] studied the influence of ribs on the transport phenomenon and flow characteristics for different Reynolds number up to $3.2 \times 103$. They concluded that the roughness causes the increase in compressive strength that is attenuated in the presence of a strong adverse pressure gradient behind each rib.

Sodjavi, et al., 2018 illustrated some photographs of the fow at dfferent high Reynolds numbers and surface roughness (smooth cylinder and G12, G6 and G24 grooved cylinders configurations). The flow structures were viusualized using microscopic iriodin particles. They concluded that for fully developed turbulent flows G12 and G24 fows have similar structural behaviour, strictly different from that of G6. They shows that the use of grooved inner cylinder increases the friction coefficient and that the groove contribution rate is a function of $\lambda / \mathrm{k}$. Within the Reynolds number range of their study (2 104 to 13104 ), the friction coefficient ratio between the rough and the smooth cylinders is approximately constant.

These studies cited above were carried out with rough structured elements that are arranged to obstruct the average flow. The surface roughness is used either by design requirement or as heat transfer promoter. They are used to be more effective in producing friction resistance than in the case of smooth surfaces.

In order to reduce the frictional drag of the skin, the rough elements aligned with the average flow constitute another category of surface roughness. In this case, the thickness of the boundary layer becomes less than the approximate height of the roughness as well as the resistance to flow becomes dominant. In this context, one can quote the studies of Hall and Joseph [10], Moradi and Floryan [11], Greidanus et al. [12] and Zhu et al. [13].

Understanding the mechanisms associated with increasing friction drag in a Taylor quilt flow in the case where the cylinder is axially grooved requires a thorough understanding of the Taylor-Couette flow.

In this work, we propose an experimental study on the hydrodynamic instabilities due to the roughness of the wall in a Couette-Taylor flow. The study was carried out with and without axial flow.

The main objective of the study is to highlight the geometric parameters effect on the flow of the flow. We mainly focused on the effect of roughness of the wall on the appearance of instabilities in the studied configuration. For this purpose, a qualitative study was performed using Kalliroscope particles to visualize the instabilities. Then, we applied polarography to different types of roughness as a quantitative study. Hence the possibility to characterize the flow regimes of the first instabilities (TVF, WVF, MWVF, and TN).

\section{EXPERIMENTAL SETUP}


A Photograph of the experimental apparatus for the study of the Taylor-Couette flow is given in Fig. 1. It consists of two vertical coaxial cylinders made of transparent Plexiglas that create a gap occupied by a fluid. The inner cylinder is driven by a $\alpha$-step motor with a variable speed.

The radii of inner and outer cylinders are ri $=88.6 \mathrm{~mm}$ and ro $=100 \mathrm{~mm}$, respectively. Thus, the gap $\mathrm{d}=\mathrm{R}_{\mathrm{o}}-\mathrm{R}_{\mathrm{i}}=11.5 \mathrm{~mm}$ and the radius ratio is $\eta=\mathrm{R}_{\mathrm{i}} / \mathrm{R}_{\mathrm{o}}=0.886$. The length of the fluid between the two cylinders $\mathrm{h}=450 \mathrm{~mm}$, which gives an axial aspect ratio of $\Gamma=\mathrm{H} / \mathrm{d}=39.47$. in Figure 1.b.

The inner-cylinder grooved configuration is presented

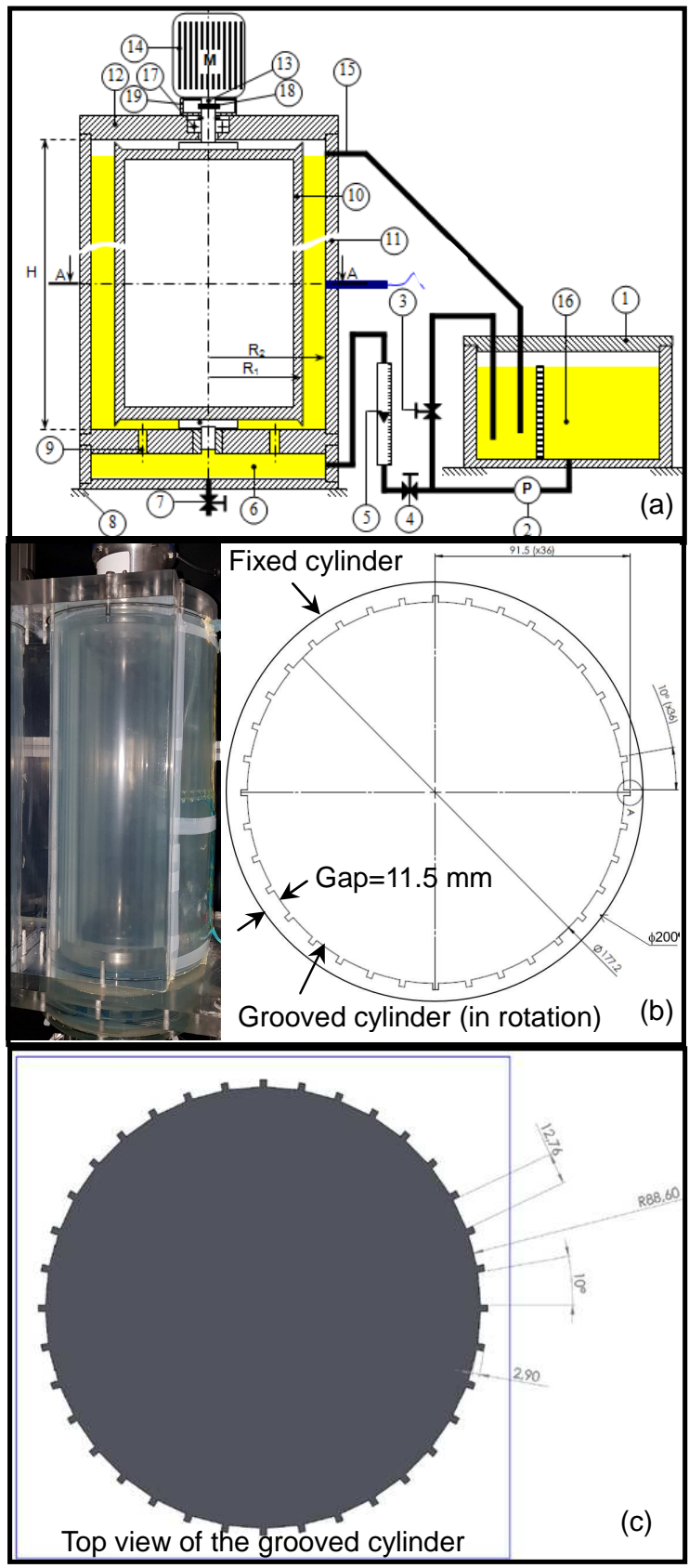

FIGURE 1: (a): SCHEMATIC VIEW OF THE CT: 1 Storage tank; 2 Pump; 3 By-pass; 4 Flowrate control valve; 5 Flowmeter; 6 CT feeder tank; 7 Drain valve; 8 Support; 9 Supply holes for the homogeneous filling of the CT gap (8 holes); $10 \mathrm{CT}$ grooved inner cylinder; $11 \mathrm{CT}$ outer cylinder; 12 Flabby; 13 Motor shaft; 14 Electrical motor; 15 Overflow; 16 Ball bearing; 17 Elastic coupling; 18 Protection; 19:

Circular probe; (b): PHOTOGRAPHY AND SKETCH WITH DIMENSIONS OF THE EXPERIMENTAL SETUP; (c):.DETAILS OF THE GROOVED INNER CYLINDER WITH DIMENSIONS.

As can be seen, the grooved cylinders are equipped with 36 identical axial rectangular cavities which are arranged periodically. They are named "Gn" where $n$ is the number of 
grooves. Our configuration corresponds to $\mathrm{Gn} 36$. The ratio of the groove width $\mathrm{w}=12.7 \mathrm{~mm}$ to its depth $\mathrm{k}=3 \mathrm{~mm}$ is equal to $\mathrm{w} / \mathrm{k}=0.9$. The ratio of the groove separation distance $\lambda=2 \Pi$ $\mathrm{r}_{\mathrm{i}} / \mathrm{n}=15.47$ to the groove depth $\mathrm{k}$ is generally used as the key parameter to distinguish the different wall roughness configurations $[14,15]$. In our configuration, this ratio $\lambda / \mathrm{k}$ correspond approximately to 5.15 for G36.

The relevant dimensionless parameter that characterizes the flow dynamic is Taylor number. It describes the relation between viscous dissipation and centrifugal force. It is defined as:

$$
T a=\frac{\Omega \cdot R_{i} \cdot d}{v} \sqrt{\frac{d}{R_{i}}}
$$

The main objective of this study is to evaluate the action of axial grooves on the behavior of Taylor-Couette flow. Thus, the grooved inner cylinder configuration is compared to the case of a Taylor Couette flow with a smooth surface. The outer cylinder has a smooth surface.

\section{II.1. Visualization of the flow by kalliroscope particles}

The Kalliroscope allows a qualitative description of the flow. It is a suspension of reflective particles. These particles are anisotropic, of dimensions $(30 \times 6 \times 0.07)$ and density of $1.62 \mathrm{~g} / \mathrm{cm} 3$. With a high index of refraction $(\mathrm{n}=1.85)$, they provide good visibility of the flow even at a low proportion.

\section{II.2. Polarography technique}

Polarography technique is an electrodiffusional method that focuses on the relationships between two conductive systems (electronic: electrodes and ionic: solution) during charge transfer composed of one or more electrons (oxidationreduction reaction). The polarography is based on the plots of Limiting Diffusion current I delivered by the probe while it is polarized by a direct voltage (between 0.5 and $0.7 \mathrm{~V}$ ) according to a given polarogramme, established function of the fluid aqueous solution characterizations and the flow regimes. The electrochemical solution that was used in our study is the ferriferrocyanide of potassium with a concentration of $25 \mathrm{~mol} / \mathrm{m}^{3}$. The choice of the indifferent inert electrolyte was done to especially limit noised diffusional limiting currents. As inert electrolyte, we used potassium sulfate $\mathrm{K}_{2} \mathrm{SO}_{4}$ at a concentration of $200 \mathrm{~mol} / \mathrm{m}^{3}$.

\section{II.3. Wall shear stress determination}

The simplifying assumptions adopted for the resolution of convection-diffusion equation generate that the Lévêque (1928) wall shear stress solution for quasi-steady, can be written as:

$$
S_{q}=S_{L e v}=\frac{D}{l^{2}}\left(\frac{S h(t)}{0.807}\right)^{3}
$$

where $S h(t)$ represents the Sherwood number, depending on the limiting current $I$, the diffusional coefficient of the electrochemical solution $D$, the Faraday number $(F=96485$ $\mathrm{C} / \mathrm{mol}$ ), the active potassium ferricyanide ion concentration $C_{0}$, the electrochemical probe width $\ell(=0.82 d$ where $d$ is the active diameter of the probe), $A$ its area and finally $n$ the number of electron exchange during the oxido-redox electrochemical reaction. This Sherwood number can be written as follow:

$$
S h=\frac{I \cdot \ell}{n \cdot F \cdot C_{0} \cdot A \cdot D}
$$

The Leveque solution becomes invalid for disturbed regime with unsteady flow. Thus, a correction of the Lévêque solution (1928) is proposed by Sobolik et al. (1987):

$$
S_{S o b}(t)=S_{q}(t)+\frac{2}{3} \theta(t)\left(\frac{\partial S_{q}(t)}{\partial t}\right)
$$

where:

$$
\theta(t)=0.486 l^{\frac{2}{3}} D^{-\frac{1}{3}} S_{q}(t)^{-\frac{2}{3}}
$$

For low Péclet numbers, the inverse method is frequently suggested. The details of the method were developed for the first time by Beck et al. (1985), who introduced it to solve the thermal transfer problems, then, by Mao and Hanratty (1990) and Maquinghem (1999) to solve the mass transfer problems.

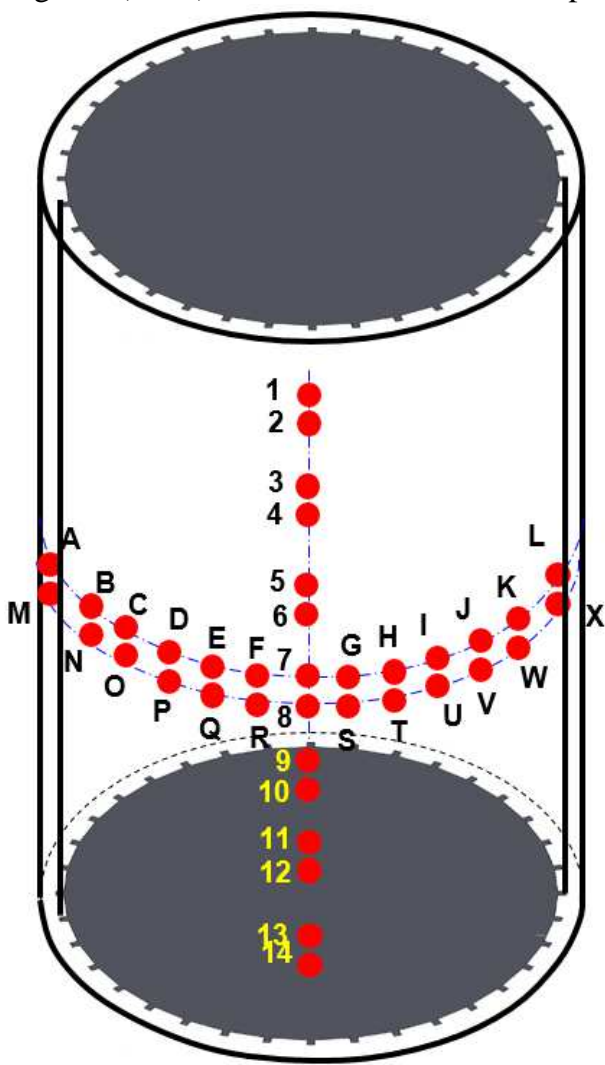

FIGURE 2: ELECTROCHEMICAL PROBES LOCALIZATION ON THE INNER WALL OF THE OUTER CYLINDER

\section{RESULTS AND DISCUSSION}

\section{III.1. Mean flow characteristics}

We investigate the effect of grooving on the general behavior of the flow. To characterize flow states in the grooved 
and smooth cases, we perform scans velocity. The question now is if roughness disturbs the flow and accelerates the transition apparition or the inverse.

To attempt to answer the question of whether the roughness makes the flux more or less stable with respect to the appearance of vortices than in the stable case, we compare the state of the flow with the case where the smooth inner cylinder.

The low dimensions of the Kalliroscope particles allow its transport by the flow. Then, the visualizations allow us to describe the flow structures and to detect the flow instabilities transitions.

\section{III.2. Taylor Vortex Flow (TVF)}

In smooth Couette-Taylor System (CTS), the first flow transition i.e. the transition from laminar Couette Flow to Taylor Vortex Flow corresponds to a critical Taylor number of 41 for A radius ratio is $\eta=\mathrm{ri} / \mathrm{ro}=0.855$ and an aspect ratio of $\Gamma=31$ (Berrich, 2011). The time-evolution of Taylor number for CTS with grooved cylinder is presented on figures 3 and 4 . It shows that the flow is steady. It corresponds thus to laminar Couette flow. For this Taylor number, we studied the timeevolution of the mass transfer

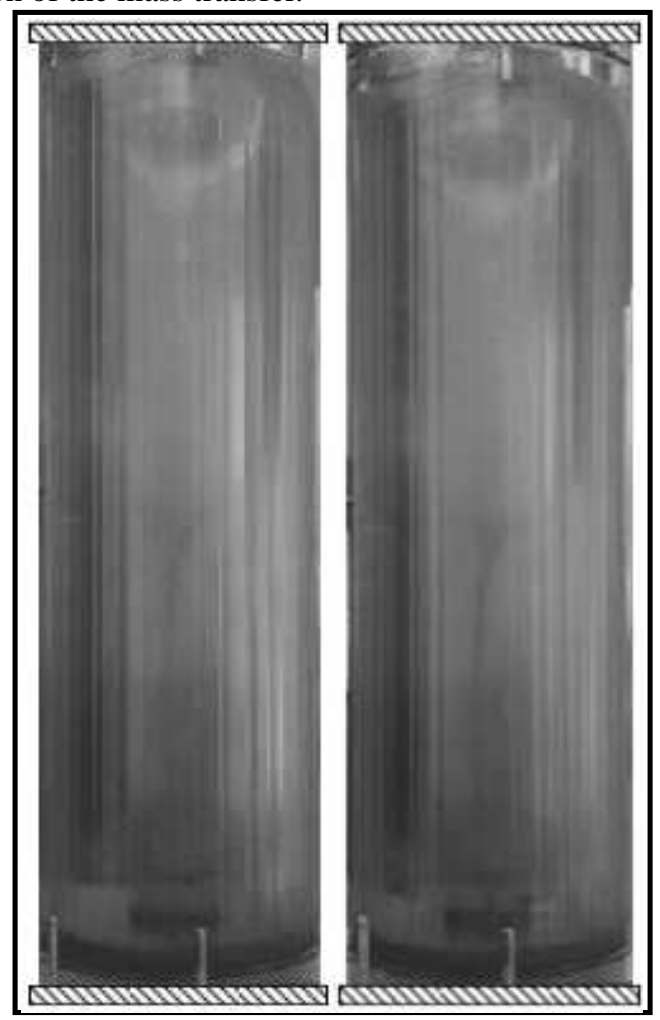

(a)

(b)

FIGURE 3: VISUALIZATION OF THE COUETTE FLOW REGIME AT: (a) $\mathrm{t}=\mathrm{t}_{0}$; (b) $\mathrm{t}=\mathrm{t}_{0}+48$

The mass transfer is almost constant (figure 5). In fact, no vortices appear for $\mathrm{Ta}=41$. To conclude, for the same Taylor number, in the case where the inner cylinder is smooth vortices appear while when the cylinder is grooved no vortices appear (figure 6). Thus, grooves relay flow transition from laminar Couette Flow to Taylor Vortex Flow.

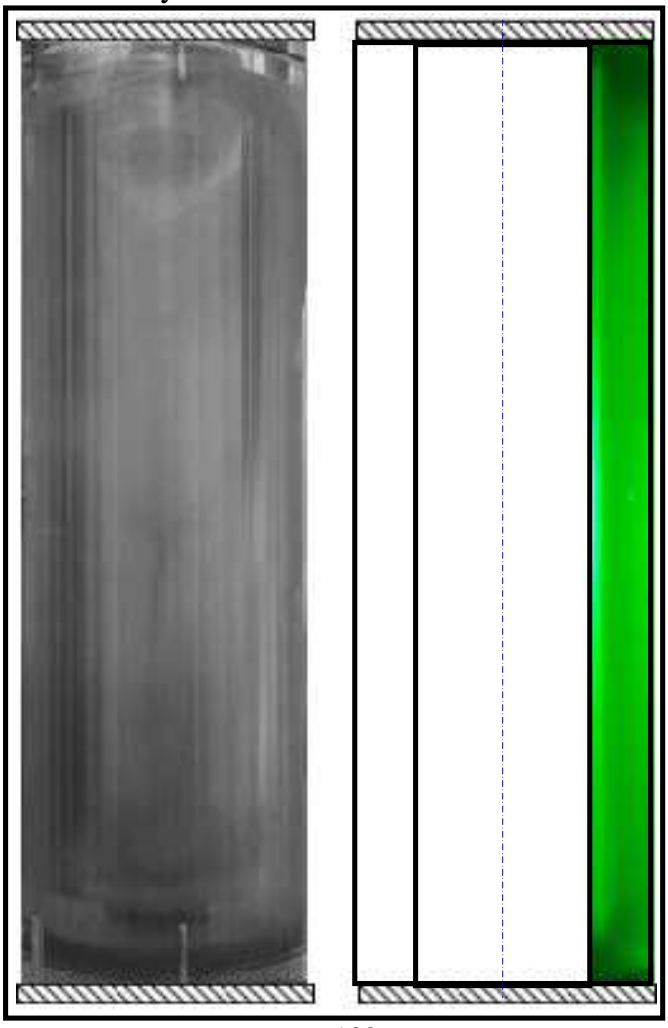

$\mathrm{t}=\mathrm{t}_{0}+132 \mathrm{~s}$

FIGURE 4: FLOW VISUALIZATION FOR THE COUETTE FLOW REGIME AT Ta $=41$

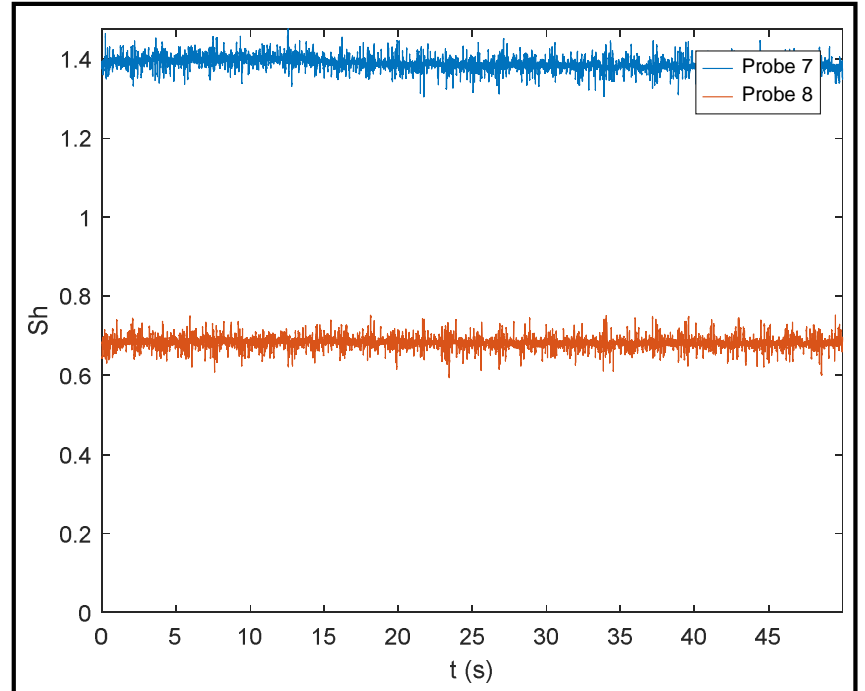

FIGURE 5: EVOLUTION OF MASS-TRANSFER RATE (SHERWOOD NUMBER Sh) FOR Ta=41

By increasing the Taylor number, the spatiotemporal evolution of the reflected intensities field becomes non homogeneous along the system. 
Figure 6 shows that shear rates are no longer constant because of the transition apparition from Couette laminar regime to Tayor Vortex regime. The average shear rate variations of the wall depend mainly on the axial and radial position at the inner wall of the outer cylinder.

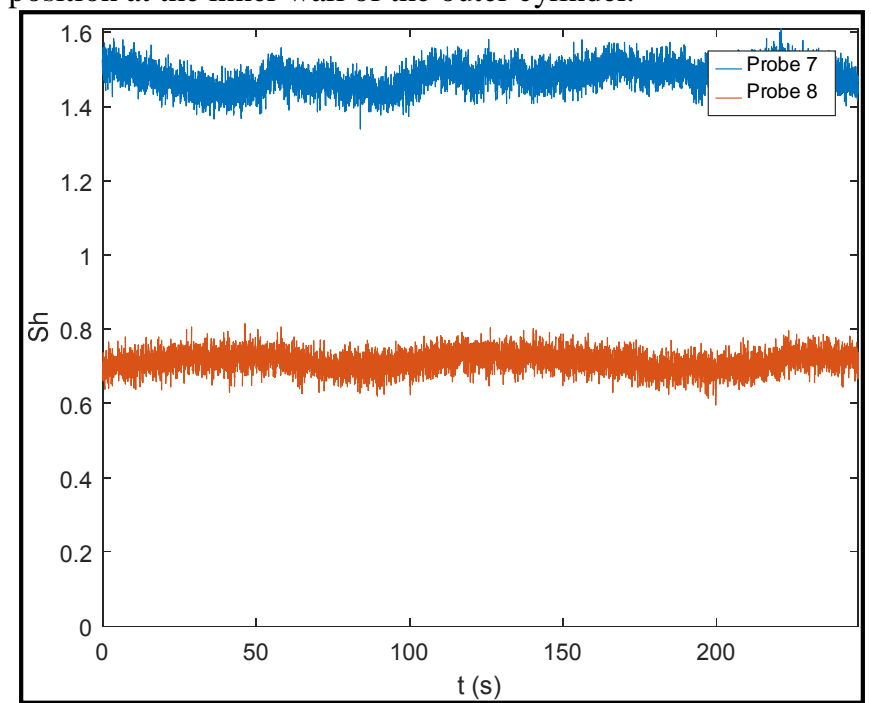

FIGURE 6: EVOLUTION OF MASS-TRANSFER RATE (Sh) FOR $\mathrm{Ta}=75$

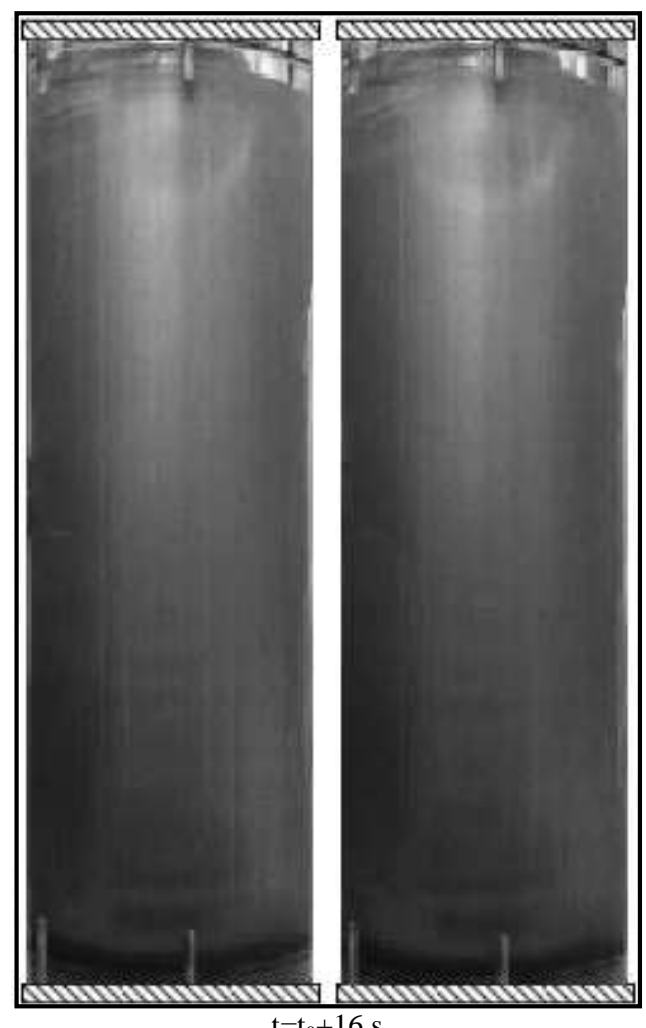

$\mathrm{t}=\mathrm{t}_{0}+16 \mathrm{~s}$

FIGURE 7: FLOW VISUALIZATION OF THE APPEARANCE OF THE FIRST TAYLOR CELLS AT Ta=75
By comparing the results in the cases where the inner cylinder is smooth or with grooves, the first vortex structures begin to develop in the same way from the ends of the latter going along the gap between the two cylinders. For cylinder with grooves, the first instabilities appear at Ta $\approx 52$ (figure 7).

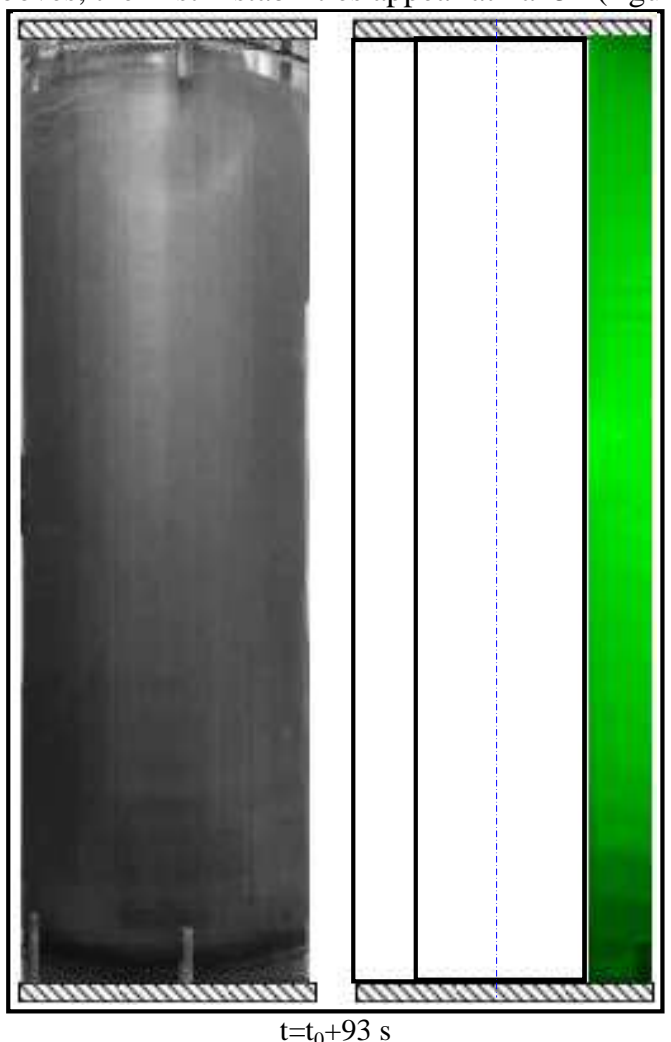

FIGURE 8: THE APPEARANCE OF THE FIRST TAYLOR CELLS

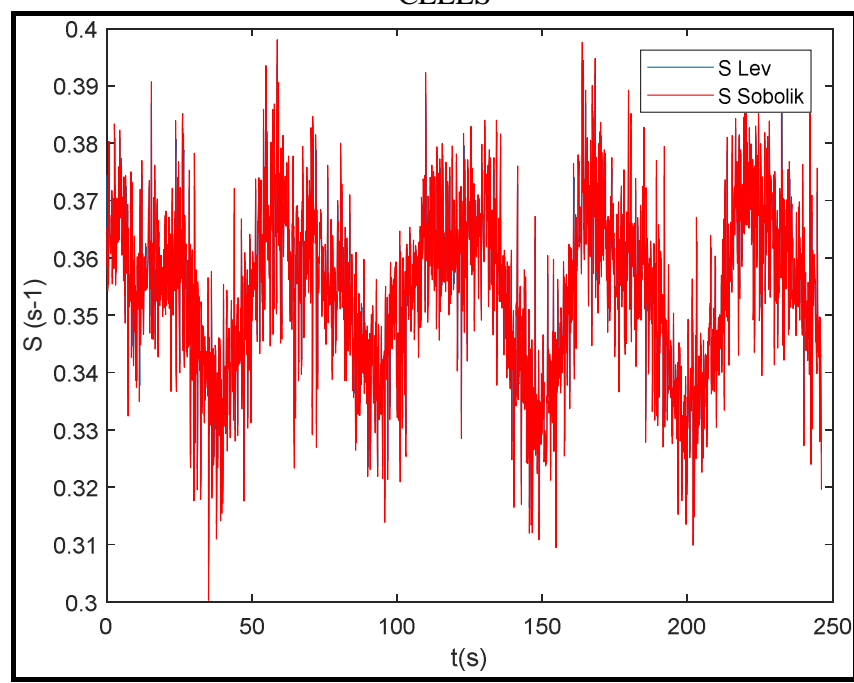

FIGURE 9: EXAMPLE OF TEMPORAL EVOLUTION OF THE PARIETAL VELOCITY GRADIENT DETERMINED FROM THE METHODS OF LEVEQUE AND SOBOLIK (1987) FOR Ta=75 (PROBE 7) 
As anticipated, the grooves produce velocity modulations and the resulting interaction with the flow breaks the primary instability responsible of Couette-Taylor roll formation, so the delay in the appearance of the first instabilities.

The evolution of the parietal velocity gradient, obtained according to the method of Levêque and sobolik (1987) as a function of time, is illustrated by the figure 9 . Note that the two signals are completely in phase.

\section{III.3. Wavy Vortex Flow (WVF)}

In order to check the dependence of the flow structure on the Roughness, Figure 10, shows the flow characteristics for the second instability.

When the Taylor number increases, a second instability appears at $\mathrm{Ta}=103$. It is characterized by the presence of wavy vortices. The evolution of the Taylor number is given in Figure 11 .

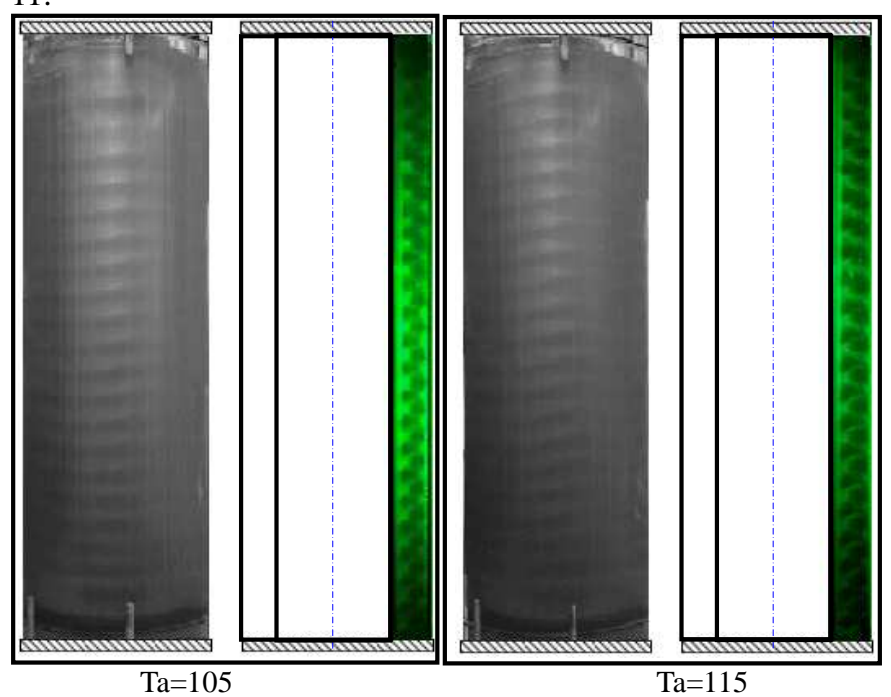

FIGURE 10: FLOW VISUALIZATION FOR THE WVF REGIME

Thus, the visualization shows that the rollers take the same periodic wave shape as in the case of smooth cylinder around the cylinder in the azimuthal and axial direction, thus breaking the axisymmetry of the rollers.

Turning to a number of Taylor $\mathrm{Ta}=105$, we notice the appearance of a new period which is superimposed on that which appeared in the previous regime (Figure 11).

The instantaneous mass transfer rate evolutions for WVF demonstrates that the wavy vortices are periodic in the axial direction with a period $\lambda$ and in the azimuthal direction with a wave number $m$ and in time with a frequency $f_{l}$.

For a radial ratio $\eta=0.87$, Edwards et al. (1991) [16] showed that the critical Taylor number corresponding to this second transition, and is equal to $\mathrm{Ta}_{\mathrm{c} 2}=234$ for 3 pairs of vortices and $\mathrm{Ta}_{\mathrm{c} 2}=52$ for 17 pairs of vortices. This may be due to the effect of the Ekman vortices that are constituted at the motionless wall of the air gap (Sobolik et al., 2011).
Figure 11 shows that in this case where we are at the beginning of the wavy Taylor vortex regime, the wave number $\mathrm{m}$ is equal to 4 .

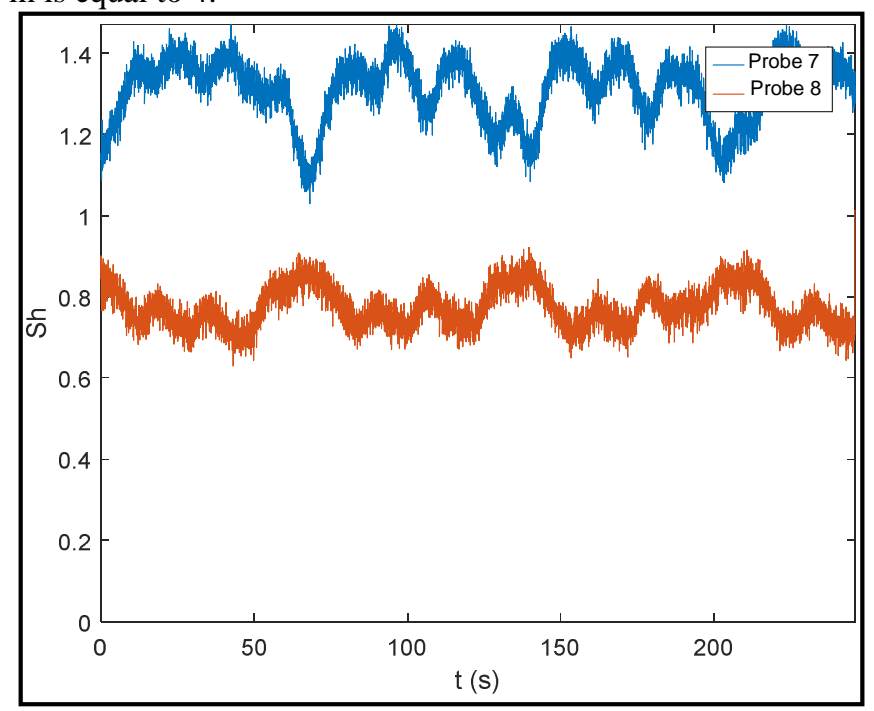

FIGURE 11: EVOLUTION OF MASS-TRANSFER RATE FOR $\mathrm{Ta}=105$

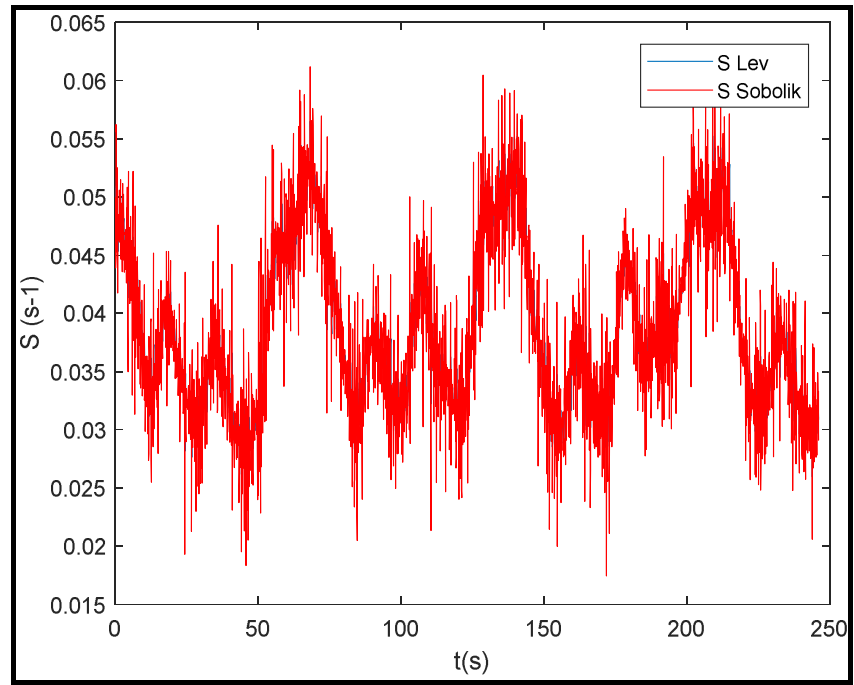

FIGURE 12: EXAMPLE OF TEMPORAL EVOLUTION OF THE PARIETAL VELOCITY GRADIENT DETERMINED FROM THE METHODS OF LEVEQUE AND SOBOLIK (1987) FOR Ta=75 (PROBE 7)

From figure 12 it can then be seen that the rapid transition from one flow regime to another results in a rapid intensification of the mass transfer and consequently of the parietal velocity gradient. This intensification is relative to the development of corrugated Taylor vortices, thus characterizing the WVF regime. The increase in the azimuthal wave causes fluctuations in the parietal velocity gradient determined by the method of Sobolik et al. (1987).

\section{III.4. Modulated Wavy Vortex Flow (MWVF)}

By further increasing the number of Taylor, the flow goes through a third transition to $\mathrm{Ta}_{\mathrm{c} 3}=142$. This transition is 
triggered when the main azimuthal wave of the WVF regime is then more homogeneous and rolls start to be unstructured (figure 13).

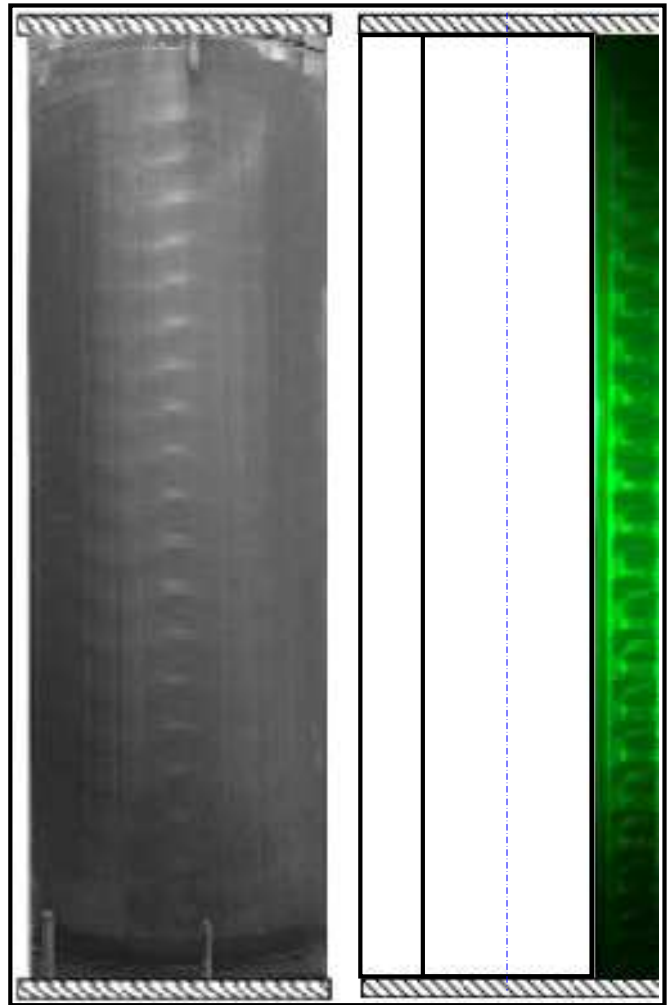

FIGURE 13: EXPERIMENTAL RESULTS FOR MWVF REGIME AT Ta $=167$

This transition is characterized by the appearance of a low frequency which present the modulation frequency of the rolls (figure 14). This regime appears with a delay compared to the case where the surface of the inner cylinder is smooth.

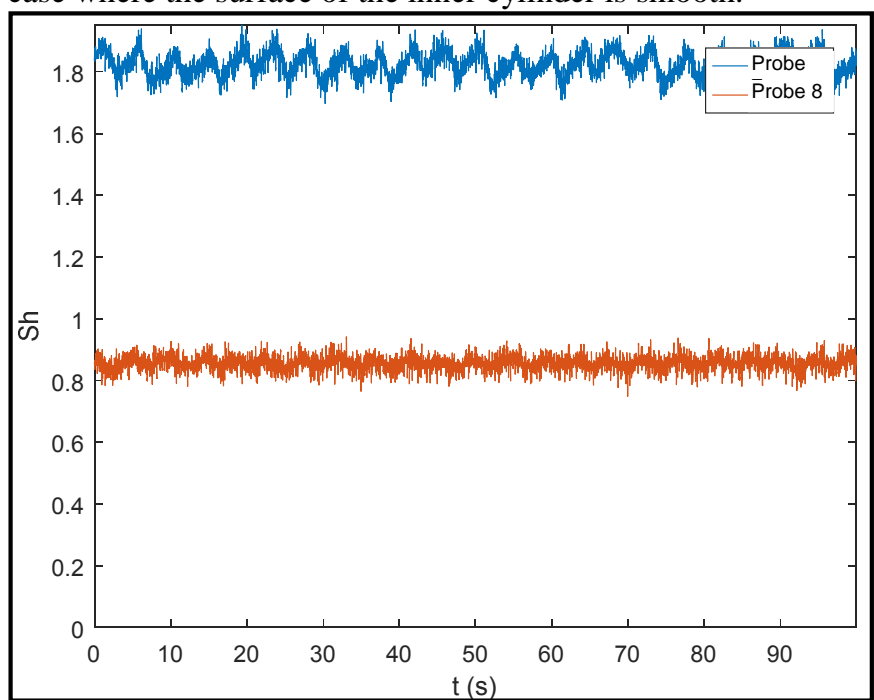

FIGURE 14: EVOLUTION OF MASS-TRANSFER RATE FOR $\mathrm{Ta}=183$

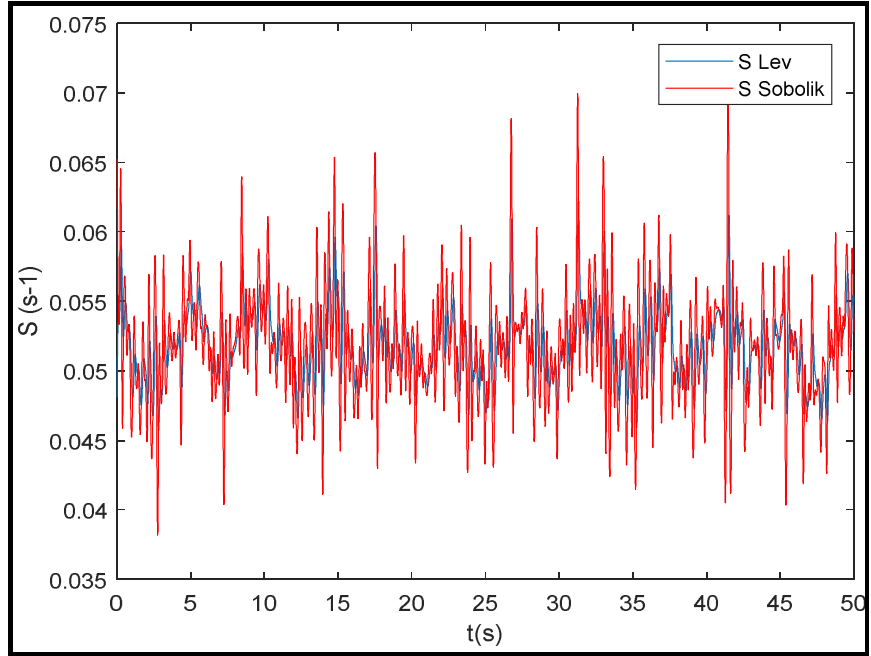

FIGURE 15: EXAMPLE OF TIME EVOLUTION OF THE WALL VELOCITY GRADIENT DETERMINED USING THE METHODS OF LEVEQUE AND SOBOLIK (1987) FOR Ta=183

The parietal velocity gradient curves obtained by the two methods of SOBOLIK and LEVEQUE (figure 15) show several oscillations. Fluctuations have become difficult to enumerate because of the superposition of different frequencies.

\section{III.5. Turbulent Taylor Vortex Flow (TTVF)}

At the instability threshold characterizing the transition between MWVF and nascent turbulent flow, the vorticity field is qualified by the homogenization of the flow in the presence of turbulent structures. This transition occurred at $\mathrm{Ta}=583$ (figure 16). It is characterized by a continuous spectrum without characteristic frequency (figure 17).

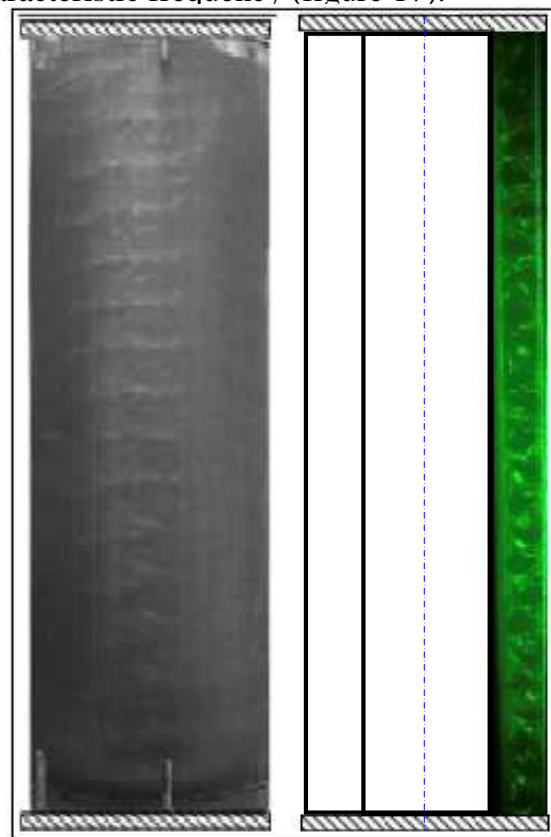

FIGURE 16: EXPERIMENTAL RESULTS FOR TTVF REGIME AT Ta $=583$ 


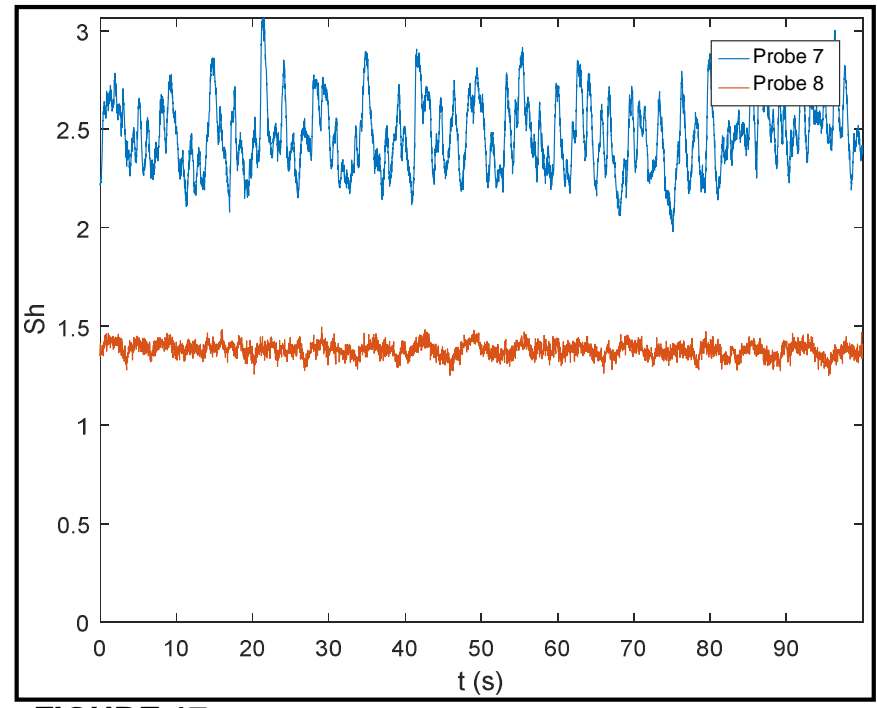

FIGURE 17: EVOLUTION OF MASS-TRANSFER RATE FOR $\mathrm{Ta}=1000$

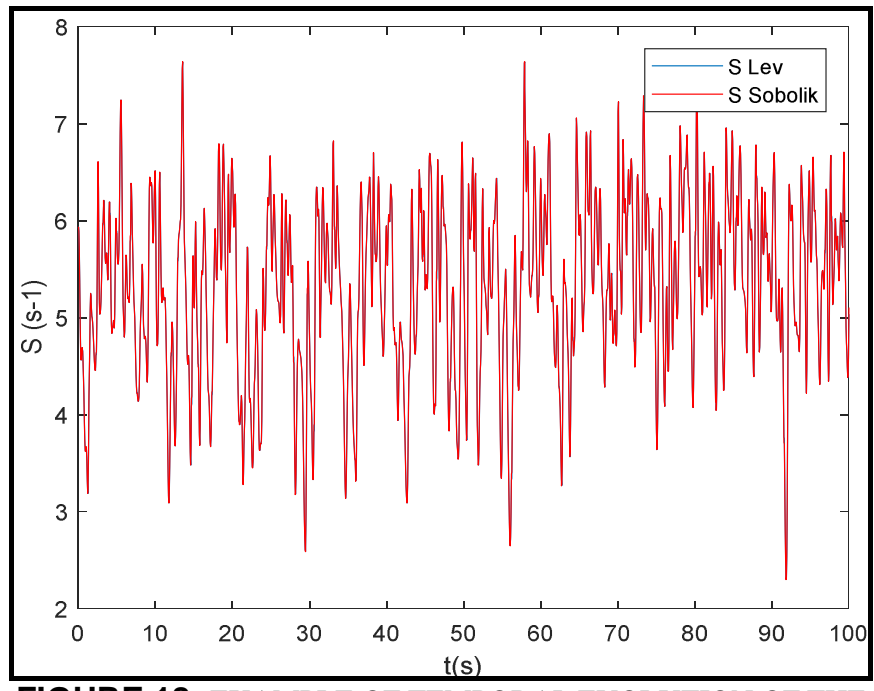

FIGURE 18: EXAMPLE OF TEMPORAL EVOLUTION OF THE PARIETAL VELOCITY GRADIENT DETERMINED FROM THE METHODS OF LEVEQUE AND SOBOLIK (1987) FOR Ta=1000

Sodjavi, et al., 2018 illustrated that for fully developed turbulent flow (high Reynolds numbers), the ratio of friction coefficient increases while increasing groove numbers (G6, G12, G24). But, they didn't study the effect of groove numbers on flow regimes instabilities. In our case G36 configuration, the turbulent flow appears at $\mathrm{Ta}=583(\mathrm{Re}=209)$ while it reaches 1210 for a smooth CT inner cylinder (Berrich, 2011).

Table 1 summarizes the different Taylor transitions corresponding to each flow regime.

Table 1. Experimental results for Critical Taylor numbers

\begin{tabular}{|c|c|c|}
\hline $\begin{array}{l}\text { Flow } \\
\text { regime }\end{array}$ & $\begin{array}{c}\text { Experimental Critical } \\
\text { Taylor number value } \\
\mathrm{Ta}_{\mathrm{c}} \text { smooth surface } \\
\text { Berrich [ 18] }\end{array}$ & $\begin{array}{c}\text { Experimental Critical } \\
\text { Taylor number value } \mathrm{Ta}_{\mathrm{c}} \\
\text { Rough surface }\end{array}$ \\
\hline TVF & 45.45 & 50 \\
\hline WVF & 60.6 & 103 \\
\hline MWVF & 606 & 142 \\
\hline TTVF & 1210 & 583 \\
\hline
\end{tabular}

\section{CONCLUSION}

This paper presents an experimental study of the roughness effect on flow behavior in a Couette-Taylor system characterized by a radius ratio $\eta=\mathrm{ri} / \mathrm{ro}=0.886$ and an axial aspect ratio of $\Gamma=\mathrm{h} / \mathrm{d}=39.47$. Two experimental techniques were used: visualizations using Kalliroscope particles and the polarography technique i.e. the Electro-Diffusion method. The spatio-temporal evolutions of the flow issued from the visualizations and the wall shear rates evolutions determined from the signal issued from the electro-diffusion probes show that surface roughness can modify the instability character of the flow: delay of the instability apparition.

For these studies, the direction of the main flow is obstructed by the ribs. Therefore, a strong pressure gradient is generated leading to increase transfer of momentum from fluid to the wall, so intense the surface friction. Then, the first instabilities appear with a delay.

\section{REFERENCES}

[1] Couette M., Etude sur le frottement des liquids, Annales de chimie et de physique 21 (1890) 433-510.

[2] [2] Diprima R.C., Eagles P.M, NG. B.S., Effect of radius ratio on the stability of Couette flow-Taylor vortex flow, Physics of Fluids, 27, 2403-2411 (1984).

[3] [3] Lueptow R.M., Docter A., Min K., Stability of axial flow in an annulus with a rotating inner cylinder, Phys. Fluids, 4, 2446 (1992).

[4] [4] Mahamdia A., Bouabdallah A., Skali S.E, Ecoulement de Taylor-Couette en géométrie finie et à surface libre, The Canadian Journal of Chemical Engineering, 83, 652-657 (2005).

[5] Rehimi F., Caractérisation expérimentale des structures tourbillonnaires derrière un cylindre en milieu confiné par PIV et par polarographie, $\mathrm{PhD}$ Thesis, University of Nantes, France (2006).

[6] Abassi W., Investigations expérimentales et modélisations numériques par la méthode de Lattice Boltzmann (LBM) pour l'étude des transferts dans les écoulements tourbillonnaires, PhD Thesis, University of Nantes, France (2014).

[7] O. Cadot, Y. Couder, A. Daerr, S. Douady, A. Tsinober, Energy injection in closed turbulent flows: Stirring 
through boundary layers versus inertial stirring, Phys. Rev. $\quad$ E $56 \quad$ (1997) 427-433, http://dx.doi.org/10.1103/PhysRevE.56.427.

[8] T.H. van den Berg, C.R. Doering, D. Lohse, D.P. Lathrop, Smooth and rough boundaries in turbulent Taylor-Couette flow, Phys. Rev. E 68 (2003) 036307, http://dx.doi.org/10.1103/PhysRevE.68.036307.

[9] T. Tsukahara, M. Ishikawa, Y. Kawaguchi, Dns study of the turbulent Taylor-vortex flow on a ribbed inner cylinder, Adv. Mech. Eng. (2013) 628490, http://dx.doi.org/ 10.1155/2013/628490.

[10] T. Hall, D. Joseph, Rotating cylinder drag balance with application to riblets, Exp. Fluids 29 (2000) 215227,http://dx.doi.org/10.1007/s003489900075.

[11] H.V. Moradi, J.M. Floryan, Flows in annuli with longitudinal grooves, J. Fluid Mech. 716 (2013) 280 315, http://dx.doi.org/10.1017/jfm.2012.547.

[12] A.J. Greidanus, R. Delfos, S. Tokgoz, J. Westerweel, Turbulent Taylor-Couette flow over riblets: drag reduction and the effect of bulk fluid rotation, Exp. $\begin{array}{llll}\text { Fluids } & 56 & \text { (2015) }\end{array}$ http://dx.doi.org/10.1007/s00348-015-1978-7.

[13] X. Zhu, R. Ostilla-Mónico, R. Verzicco, D. Lohse, Direct numerical simulation of Taylor-Couette flow with grooved walls: torque scaling and flow structure, J. Fluid Mech. 794 (2016) 746-774, http://dx.doi.org/10.1017/jfm.2016.179.

[14] M. Motozawa, T. Ito, K. Iwamoto, H. Kawashima, H. Ando, T. Senda, Y. Tsuji, Y. Kawaguchi, Experimental investigations on frictional resistance and velocity distribution of rough wall with regularly distributed triangular ribs, International Journal of Heat and Fluid Flow, $41 \quad$ (2013) 112-121, doi:10.1016/j. ijheatfluidflow.2013.03.004.

[15] Tsukahara T., Ishikawa M., Kawaguchi Y., DNS study of the turbulent Taylor-Vortex on a ribbed inner cylinder, Advances in Mech. Eng., (2013), https://doi.org/10.1155/2013/628490.

[16] Edwards W.S., Beane S.R, Varma S., Onset of wavy vortices in the finite-length Couette-Taylor problem, Phys. Fluids, Vol. 3 (1991).

[17] Sobolik V., Jirout T., Havlica J., Kristiawan M.,Wall Shear Rates in Taylor Vortex Flow, Journal of Applied Fluid Mechanics", Vol. 4, (2), 25-31, (2011).

[18] Berrich E., «Analyse expérimentale des interactions écoulement - paroi par PIV et polarographie en utilisant des sondes multi-segmentées : Application à un rhéomètre plan-plan et un système de CouetteTaylor », PhD Thesis, University of Nantes, France (2006). 